\title{
CORRECTION
}

\author{
Salvatore Federico • Alfio Grillo • Reuven Segev
}

\section{Correction to: Material description of fluxes in terms of differential forms}

Dedicated to Prof. David Steigmann in recognition of his contributions

Published online: 1 August 2018

(C) Springer-Verlag GmbH Germany, part of Springer Nature 2018

Correction to: Continuum Mech. Thermodyn. (2016) 28:379-390

https://doi.org/10.1007/s00161-015-0437-2

Although the final result presented in Equation (34) of our work [1] is correct, the proof of Equation (34) contains an error. The text starting immediately after Equation (33) with "When a metric tensor $\boldsymbol{g}$..." and ending immediately before Equation (36) with "...in the alternative notation" should be replaced with the text below.

\section{Correction of the Proof of Equation (34)}

Let us assume that the space $\mathcal{S}$ is equipped with a metric tensor $\boldsymbol{g}$, i.e., a symmetric and positive-definite tensor field valued in $[T S]_{2}^{0}$, defining the scalar product of two vectors $\boldsymbol{u}$ and $\boldsymbol{v}$ as $\boldsymbol{u} \cdot \boldsymbol{v}=\boldsymbol{g}(\boldsymbol{u}, \boldsymbol{v})$. The metric $\boldsymbol{g}$ induces the musical isomorphisms $b: T \mathcal{S} \rightarrow T^{\star} \mathcal{S}: \boldsymbol{v} \mapsto b(\boldsymbol{v}) \equiv \boldsymbol{v}^{b}$, which maps a vector $\boldsymbol{v}$ with components $v^{c}$ to a covector $\boldsymbol{v}^{b}$ with components $g_{a c} v^{c}$, and its inverse $\sharp: T^{\star} \mathcal{S} \rightarrow T \mathcal{S}: \boldsymbol{\alpha} \mapsto \sharp(\boldsymbol{\alpha}) \equiv \boldsymbol{\alpha}^{\sharp}$, which maps a covector $\boldsymbol{\alpha}$ with components $\alpha_{c}$ to a vector $\boldsymbol{\alpha}^{\sharp}$ with components $g^{a c} \alpha_{c}$, where $g^{a c}$ are the components of the inverse of the matrix $\llbracket g_{a b} \rrbracket$ of $\boldsymbol{g}$. The isomorphism $\sharp$ and the metric tensor $\boldsymbol{g}$ induce the scalar product of covectors $\alpha . \beta=g\left(\alpha^{\sharp}, \beta^{\sharp}\right)=\alpha\left(\beta^{\sharp}\right)$.

The $(n-1)$-dimensional tangent bundle $T s$ of the hypersurface $s$ determines a 1-dimensional sub-bundle of $T^{\star} \mathcal{S}$ containing the annihilators of $T$ s, i.e., the covectors $\boldsymbol{v}$ such that $\boldsymbol{v} \boldsymbol{u} \equiv \boldsymbol{v}(\boldsymbol{u})=0$, for every $\boldsymbol{u}$ in $T s$. Moreover, using the scalar product of covectors, we can define the unit normal covector $\boldsymbol{n}$ to the hypersurface $s$ as the annihilating covector such that $\|\boldsymbol{n}\|^{2}=\boldsymbol{n} . \boldsymbol{n}=1$.

The integral (33) of an ( $n-1)$-form $\omega$ on the hypersurface $s$ can be expressed in terms of the axial vector field $\boldsymbol{w}$ of $\boldsymbol{\omega}$ with respect to the volume form $\mu$, i.e., $\boldsymbol{w}$ is such that $\iota_{\boldsymbol{w}} \boldsymbol{\mu}=\boldsymbol{\omega}$. If we introduce the axial projector $\boldsymbol{a}=\boldsymbol{n}^{\sharp} \otimes \boldsymbol{n}$ (in components, $a_{b}^{a}=n^{a} n_{b}$ ) and the transverse projector $\boldsymbol{t}=\boldsymbol{i}-\boldsymbol{n}^{\sharp} \otimes \boldsymbol{n}$ (in components,

The original article can be found online at https://doi.org/10.1007/s00161-015-0437-2.

S. Federico $(\varangle)$

Department of Mechanical and Manufacturing Engineering, The University of Calgary, 2500 University Drive NW, Calgary, AB T2N1N4, Canada

E-mail: salvatore.federico@ucalgary.ca

A. Grillo

DISMA - Department of Mathematical Sciences “G.L. Lagrange”, Politecnico di Torino, Corso Duca degli Abruzzi 24, 10124 Torino, Italy

R. Segev

Department of Mechanical Engineering, Ben Gurion University, P.O. Box 653, 84105 Beer-Sheva, Israel 
$\left.t^{a}{ }_{b}=\delta^{a}{ }_{b}-n^{a} n_{b}\right)$, where $\boldsymbol{i}$ is the spatial identity tensor, it holds that $\boldsymbol{i}=\boldsymbol{a}+\boldsymbol{t}$ and that any vector field $\boldsymbol{w}$ can be decomposed as

$$
w=i w=(a+t) w=a w+t w=w_{a}+w_{t},
$$

where $\boldsymbol{w}_{a}=\boldsymbol{a} \boldsymbol{w}=(\boldsymbol{n} \boldsymbol{w}) \boldsymbol{n}^{\sharp}$ and $\boldsymbol{w}_{t}=\boldsymbol{t} \boldsymbol{w}=\boldsymbol{w}-(\boldsymbol{n} \boldsymbol{w}) \boldsymbol{n}^{\sharp}$ are the axial component and the transverse component of $\boldsymbol{w}$, respectively. By construction, $\boldsymbol{w}_{t}$ is an element of the tangent bundle of the $(n-1)$-dimensional manifold $s \subset \mathcal{S}$. Hence, due to linearity, the $(n-1)$-form $\omega=\iota_{w} \boldsymbol{\mu}$ can be written as

$$
\omega=\iota_{w} \mu=\iota_{\left(w_{a}+w_{t}\right)} \boldsymbol{\mu}=\iota_{w_{a}} \boldsymbol{\mu}+\iota_{w_{t}} \mu .
$$

Let now $\left\{\boldsymbol{u}_{1}, \ldots, \boldsymbol{u}_{n-1}\right\} \subset T s$ be a set of linearly independent vectors spanning $T s$. Since $\boldsymbol{w}_{t}$ can be expressed as a linear combination of $\boldsymbol{u}_{1}, \ldots, \boldsymbol{u}_{n-1}$, we obtain

$$
\left(\boldsymbol{\iota}_{\boldsymbol{w}_{t}} \boldsymbol{\mu}\right)\left(\boldsymbol{u}_{1}, \ldots, \boldsymbol{u}_{n-1}\right)=\boldsymbol{\mu}\left(\boldsymbol{w}_{t}, \boldsymbol{u}_{1}, \ldots, \boldsymbol{u}_{n-1}\right)=0 .
$$

Comparing Eq. (C3) with the definition of $\omega$ in Eq. (C2), we find

$$
\boldsymbol{\omega}\left(\boldsymbol{u}_{1}, \ldots, \boldsymbol{u}_{n-1}\right)=\left(\boldsymbol{\iota}_{\boldsymbol{w}} \boldsymbol{\mu}\right)\left(\boldsymbol{u}_{1}, \ldots, \boldsymbol{u}_{n-1}\right)=\left(\boldsymbol{\iota}_{\boldsymbol{w}_{a}} \boldsymbol{\mu}\right)\left(\boldsymbol{u}_{1}, \ldots, \boldsymbol{u}_{n-1}\right),
$$

and, since (C4) must hold true for all $(n-1)$-tuples $\left\{\boldsymbol{u}_{1}, \ldots, \boldsymbol{u}_{n-1}\right\} \subset T s$, we can write

$$
\omega=\iota_{w} \mu \equiv \iota_{w_{a}} \mu,
$$

i.e., only the axial component of $\boldsymbol{w}$, which is the component parallel to the vector $\boldsymbol{n}^{\sharp}$ associated with the normal covector $\boldsymbol{n}$ to the hypersurface $s$, contributes to $\boldsymbol{\omega}$. Finally, by exploiting the result $\boldsymbol{w}_{a}=(\boldsymbol{n} \boldsymbol{w}) \boldsymbol{n}^{\sharp}=(\boldsymbol{w n}) \boldsymbol{n}^{\sharp}$ and the linearity of the interior product, Eq. (C5) becomes

$$
\omega=\iota_{w} \mu \equiv \iota_{w_{a}} \mu=(w n) \iota_{n^{\sharp}} \boldsymbol{\mu}=(w n) \boldsymbol{\alpha},
$$

where

$$
\alpha=\iota_{n^{\sharp}} \mu
$$

is the ( $n-1)$-form induced on the hypersurface $s$ by the volume form $\mu$ and the metric $g$. Therefore, on the basis of these results, the flux of an extensive quantity $q$ across the hypersurface $s$ can be expressed in the alternative notation $[\ldots]$

\section{Reference}

1. Federico, S., Grillo, A., Segev, R.: Material description of fluxes in terms of differential forms. Continuum Mech. Thermodyn. 28, 379-390 (2016). https://doi.org/10.1007/s00161-015-0437-2

Publisher's Note Springer Nature remains neutral with regard to jurisdictional claims in published maps and institutional affiliations. 